\title{
Non-invasive Biomarkers of Idiopathic Pulmonary Fibrosis and Lung Cancer
}

Martina Armati, MD, Olivia Michalczyszyn, MD, Katarzyna Zaczyk, MD, and Siena IPF team

\section{ABSTRACT}

Introduction: The aim of this article is to collect and summarize the evidence related to the role of non-invasive biomarkers in lung cancer and idiopathic pulmonary fibrosis (IPF), to better understand the point in common of these two conditions and the possible uses, and to better understand and define these diseases individually and together. Methods: The methodology for this study consisted of a systematic review searching on PubMed covering the period between 2000 and 2020. From this research, only 13 articles were considered as most relevant regarding this theme. Conclusion: This review provides a current state of knowledge regarding the use of non-invasive biomarkers in lung cancer and IPF to address clinical practice and research proposals to better understand the correlation between IPF and lung cancer and to give other possible information about the severity and the prognosis in IPF patients. (BRN Rev. 2021;7(2):109-14)

Corresponding author: Martina Armati, martina.armati@gmail.com

Keywords: Biomarkers. Idiopathic pulmonary fibrosis. Interstitial lung disease. Lung cancer. Non-invasive biomarkers. Peripheral biomarkers. 


\section{INTRODUCTION}

Idiopathic pulmonary fibrosis (IPF) is defined as a specific chronic progressive interstitial lung disease (ILD) of unknown cause, confined to the lung. It generally occurs in older ex-smoker adults. Its pathogenesis shares many similarities with lung cancer, particularly in relation to the molecular pathways as well as many genetic and cell processes ${ }^{1}$. Including the aberrant expression of miRNAs regulating non-small cell lung cancer (NSCLC) and IPF or the crucial role of tyrosine kinase inhibitor directed against growth factors (platelet-derived growth factor, vascular endothelial growth factor and fibroblast growth factor) involved in the pathogenesis of both lung diseases $^{1,2}$.

Several studies have reported a high prevalence of lung cancer in patients with IPF' ${ }^{2}$ IPF patients have an increased risk of developing lung cancer compared to the general population ${ }^{3}$. In particular, lung cancer occurs in the peripheral areas of the lower lobes, where fibrosis is predominant and from honeycombing areas or in the border between honeycombing and non-fibrotic areas ${ }^{4}$. The association of IPF and lung cancer is related to a negative prognosis.

The pharmacological antifibrotic treatment of IPF includes antineoplastic therapies, like nintedanib. Nintedanib is a tyrosine-kinase inhibitor, firstly developed as an anticancer antiangiogenetic drug and now approved for the treatment of IPF ${ }^{5}$.

IPF risk factors are similar to those of lung cancer, including smoking, pollution or other environmental and professional exposure at old age ${ }^{6}$.
The biomarker research is developed in oncology to define the diagnosis, the patient's prognosis and treatment response as well as for treatment selection. Unfortunately, there is no single reliable biomarker to be used in the clinical practice for IPF diagnosis and prognosis or for early detection of lung cancer development in IPF patients. The early diagnosis of neoplasms in these patients is principally related to computed tomography (CT) periodical follow-up 7 .

This review aims to describe the most promising non-invasive biomarkers, common in both lung cancer and IPF patients.

\section{PERIPHERAL BIOMARKERS OF IPF AND LUNG CANCER}

- Carcinoembryonic Antigen (CEA) is a glycoprotein involved in cell adhesion and produced during foetal development. In physiological conditions, the production of this protein ceases after birth. It is used as a tumor marker in particular for gastrointestinal and lung cancers, even if its increase may be associated also with non-malignant diseases, like cirrhosis and pancreatitis ${ }^{8}$. Cigarette smoking can be associated with elevated serum CEA levels reflecting smokeinduced dysplasia or metaplasia of respiratory epithelium?.

Elevated serum and bronchoalveolar levels of CEA have been described in IPF patients ${ }^{10}$ and it has been demonstrated that this protein is expressed inside the areas of lung epithelial metaplasia ${ }^{11}$. In the study of Hao $\mathrm{C}$ et al. ${ }^{12}$, it was suggested that increased serum CEA levels might be an indicator of tissue 
TABLE 1. Collection of the most relevant manuscripts of biomarkers of IPF and lung cancer

\begin{tabular}{|c|c|c|c|}
\hline Reference & Biomarker(s) studied & Condition & Sample \\
\hline Hao C et al. $(2019)^{12}$ & CEA & Lung cancer & Serum \\
\hline Moll SA et al. $(2020)^{15}$ & CA $15-3$ & IPF & Serum \\
\hline Vercauteren IM et al. (2015) ${ }^{17}$ & CYFRA 21.1 & IPF & BAL \\
\hline Miyazaki K et al. $(2010)^{18}$ & $\mathrm{KL}-6$ & Lung cancer & Serum \\
\hline Yoshimasu T et al. $(2012)^{20}$ & $\mathrm{KL}-6$ & Lung cancer & Serum \\
\hline Balestro $E$ et al. $(2020)^{26}$ & CA 19-9 & IPF & Serum \\
\hline Mauro $C$ et al. $(2019)^{29}$ & CEA, CYFRA 21-1, ProGRP, NSE & $\begin{array}{l}\text { Lung cancer } \\
\text { (small-cell lung cancer) }\end{array}$ & Serum \\
\hline Hara A et al. $(2012)^{32}$ & S100A9 & IPF & BALF \\
\hline Koh HM et al. $(2019)^{33}$ & S100A9 & $\begin{array}{l}\text { Lung cancer } \\
\text { (non-small-cell lung carcinoma) }\end{array}$ & Tissue \\
\hline Oremek GM et al. $(2007)^{34}$ & NSE, CEA, ProGRP, CYFRA 21-1, CRP, TNF alpha & Lung cancer & Serum \\
\hline Dai H et al. $(2014)^{35}$ & CEA, CA125, CA19-9, NSE & IPF, lung cancer & Serum \\
\hline Liu Y et al. $(2018)^{36}$ & CEA, CA125 & IPF, lung cancer & Serum \\
\hline Bennett D et al. $(2019)^{37}$ & S100A9, KL-6 & IPF & BAL \\
\hline
\end{tabular}

BAL: bronchoalveolar lavage; BALF: bronchoalveolar lavage fluid; CA: carbohydrate antigen; CEA: carcinoembryonic antigen; IPF: idiopathic pulmonary fibrosis; KL: Krebs von den Lungen; TNF-alfa: tumour necrosis factor-alpha.

epithelium damage, caused by ILDs. CEA was proposed as a biomarker of both IPF and lung cancer.

- Carbohydrate antigen 15-3 (CA 15-3): this soluble form of MUC-1 protein is the most widely used serum marker in patients with breast cancer ${ }^{13}$. This protein is also increased in IPF and in sarcoidosis patients, mainly in phase IV with lung fibrotic involvement. CA 15-3, together with Cytokeratin 19, reveals the fibroblast activities as they are expressed by fibroblast foci and from cell cultures of lung fibroblasts of IPF lungs ${ }^{14}$. The study by Moll et al. ${ }^{15}$ has indicated that CA 15-3 levels are higher in IPF patients compared to sarcoidosis patients. The advantage of this marker, as all the others blood markers, is that the measurement is low risk and can be easily obtained. Also, its cost is decreased compared to e.g., Krebs von den Lungen 6 (KL-6) measurements.

- CYFRA 21-1: a polypeptide that recognizes a soluble cytokeratin 19 fragment; it is released from epithelial cells upon cell death. This polypeptide is not specific to lung cancer and it has been associated with inflammation. For this reason, CYFRA 21-1 has been investigated as a marker for epithelial damage in chronic inflammatory diseases including IPF. It has been shown that its serum and bronchoalveolar lavage (BAL) levels are elevated in IPF patients with poor prognosis ${ }^{16,17}$.

- KL-6: this high molecular weight mucinlike glycoprotein is a biomarker of both 
ILD and lung cancer ${ }^{18}$. A murine IgG1 monoclonal antibody was developed to recognize a sialylated sugar chain, a high molecular weight $(200 \mathrm{kDa})$ glycoprotein designated KL-6, by immunizing a mouse with human lung adenocarcinoma cells ${ }^{19}$. KL-6 was first suggested as a serum biomarker for lung, breast and pancreatic cancers. In lung cancer, its serum levels could indicate malignancy progression ${ }^{20}$. However, KL-6 showed a lower diagnostic accuracy than other tumor markers, such as carcinoembryonic antigen, a reliable predictor of treatment response in NSCLC ${ }^{21}$. KL-6 could also be considered as a sensitive biomarker of IPF, useful to predict the outcome and the treatment response. It has been widely studied and it is over-expressed in alveolar epithelial cells of ILD patients ${ }^{22}$. In those patients, KL-6 is mainly related to fibrotic involvement while tumor marker proteins cannot differentiate neoplastic from fibrotic lung disorders, being increased in both these conditions ${ }^{23,24}$.

- Carbohydrate antigen 19-9 (CA 19-9): this glycoprotein produced by epithelium is a common gastrointestinal, mainly pancreatic, tumor biomarker. Its elevated levels in serum, have also been observed in benign respiratory diseases such as IPF and ILDs in general ${ }^{25-27}$.

- Neuron-specific enolase (NSE): increased levels of this biomarker are expressed in brain, neurons and neuroendocrine cells. It has been shown to be an effective serum biomarker inside the areas of neuronal damage. Elevation of NSE levels has been observed in patients affected with neuroendocrine tumors such as small-cell lung cancer $(\mathrm{SCLC})^{28}$, although its sensitivity is quite low $^{30}$. Moreover, its role in other ILDs has to be investigated, but a recent study from D'Alessandro et al. ${ }^{30}$ has shown how NSE levels in IPF patients were higher compared to non-IPF patients ${ }^{30}$.

- Calgranulin B (S100A9) is a member of S100 family of calcium-binding proteins. They are present in various cell types including neutrophils. Its activity is expressed via two receptors: the toll-like receptor 4 (TLR4) and the receptor for advanced glycation end products (RAGE). This protein stimulates pro-inflammatory responses ${ }^{31}$. Increased levels of this marker have been reported in BAL fluid of IPF patients comparing to other fibrotic interstitial pneumonias, including non-specific interstitial pneumonia (I-NSIP), collagen vascular disease-related interstitial pneumonia (CVD-IP) and healthy individuals, revealing that S100A9 might be a useful marker in the clinical setting $^{32}$. It was also noted that high S100A9 levels are associated with several cancer types, including non-small-cell lung carcinoma. Its expression is also higher in cancer tissues compared to para-cancer tissues of NSCLC patients and is related to the degree of tumor differentiation. On this basis, a recent manuscript speculated that S100A9 could be used as a potential molecular marker for NSCLC diagnosis and prognosis $^{33}$. Calgranulin B plays several immunological functions being mainly involved in chronic inflammation and cancer. It can participate in the recruitment of neutrophils and leukocytes in inflamed tissue, oxidant/ antioxidant balance, adhesion of neutrophils to fibronectin, and regulation of apoptosis. In a proteomic study, calgranulin B was 
found up-regulated in the BAL of patients with IPF with respect to controls and patients with other ILD. The immunohistochemistry, done in a subgroup of patients with IPF, revealed a patchy distribution of calgranulin $\mathrm{B}$, predominantly around areas of fibrotic remodeling. Calgranulin B may be a trigger molecule involved in the evolution and progression of IPF, being overexpressed in BAL of patients with IPF with severe functional deterioration and in the peribronchiolar area bordering zones of honeycombing.

\section{PANEL OF BIOMARKERS}

There are several biomarkers which levels are altered both in IPF and lung cancer and these bioindicators could be used to study and evaluate both diseases. Many of them are worth further investigation. Oremek et al. ${ }^{34}$ have investigated several cancer and inflammatory markers: NSE, CEA, serum pro-gastrin releasing peptide (ProGRP), CYFRA 21-1, C-reactive protein (CRP) and tumour necrosis factor-alpha (TNF alpha) in order to test their clinical and prognostic usefulness. The authors showed that elevated levels of CYFRA-21-1, NSE and ProGRP can be associated with lung cancer progression and that the combination of a panel of biomarkers can improve prognostic power. In this way, the study done by Dai H. et al. ${ }^{35}$ investigated serum tumour marker levels in different types of ILD and in ILD combined with lung cancer (ILD-CA), to define the relationship between serum tumour marker levels and ILD.

The authors observed that serum CEA and CA125 biomarker levels were higher in patients affected by ILD than in controls; moreover, serum CEA, CA125, CA19-9 and NSE were furtherly elevated in patients with ILD associated with lung neoplasms. Subtype analysis revealed that serum CEA, CA19-9 and CA125 levels were higher in patients with IPF. Based on these outcomes, researchers suggested that CEA, CA19-9 and CA125 tumor markers not only increase the likelihood of cancer but they are also increased in fibrosis and may indicate a future cancer risk.

In another interesting study ${ }^{31,36}$, the authors show that both CEA and CA125 were highly elevated in IPF patients with lung cancer compared to patients with IPF and no lung neoplasms. Both the serological biomarkers, together with chest high-resolution CT (HRCT) features were suggested as bioindicator of lung cancer development risk in IPF patients.

Bennett et al. ${ }^{37}$ investigated two biomarkers, Calgranulin B and KL-6, in BAL fluid of IPF patients. The researchers state that both these biomarkers were reliable indicators of IPF prognosis, and the combination of both proteins could help discriminating patients with severe or advanced disease from early phase IPF patients.

\section{CONCLUSION}

The aim of this narrative review is to provide a current state of knowledge regarding the use of non-invasive biomarkers in lung cancer and IPF, to address clinical practice and research proposals, to better understand the correlation between IPF and lung cancer, and to give other possible information about the severity and the prognosis in IPF patients. 


\section{DISCLOSURES}

\section{Dr. Michalczszyn, Dr. Zaczyk, and Dr. Arma- ti have nothing to disclose.}

\section{REFERENCES}

1. American Thoracic Society. Idiopathic pulmonary fibrosis: diagnosis and treatment. International consensus statement. American Thoracic Society (ATS), and the European Respiratory Society (ERS). Am J Respir Crit Care Med. 2000;161:646-64.

2. Matsushita H, Tanaka S, Saiki $Y$ et al. Lung cancer associated with usual interstitial pneumonia. Pathol Int. 1995;45:925-32.

3. Daniels CE, Jett JR. Does interstitial lung disease predispose to lung cancer? Curr Opin Pulm Med. 2005;11:431-7.

4. Khan KA, Kennedy MP, Moore E et al. Radiological characteristics, histological features and clinical outcomes of lung cancer patients with coexistent idiopathic pulmonary fibrosis. Lung. 2015;193:71-7.

5. Richeldi L, Cottin V, Flaherty KR et al. Design of the INPULSISTM trials: two phase 3 trials of nintedanib in patients with idiopathic pulmonary fibrosis. Respir Med. 2014;108:1023-30.

6. Selman M, King TE, Pardo A, American Thoracic Society, European Respiratory Society, American College of Chest Physicians. Idiopathic pulmonary fibrosis: prevailing and evolving hypotheses about its pathogenesis and implications for therapy. Ann Intern Med. 2001;134:136-51.

7. Kammer MN, Massion PP. Noninvasive biomarkers for lung cancer diagnosis, where do we stand? J Thorac Dis. 2020;12:3317-30.

8. Goldstein MJ, Mitchell EP. Carcinoembryonic antigen in the staging and follow-up of patients with colorectal cancer. Cancer Invest. 2005;23:338-51.

9. Fukuda I, Yamakado M, Kiyose H. Influence of smoking on serum carcinoembryonic antigen levels in subjects who underwent multiphasic health testing and services. J Med Syst. 1998;22:89-93.

10. Takahashi H, Nukiwa T, Matsuoka R et al. Carcinoembryonic antigen in bronchoalveolar lavage fluid in patients with idiopathic pulmonary fibrosis. Jpn J Med. 1985;24:236-43.

11. Fahim A, Crooks MG, Wilmot R, Campbell AP, Morice AH, Hart SP. Serum carcinoembryonic antigen correlates with severity of idiopathic pulmonary fibrosis. Respirol Carlton Vic. 2012;17:1247-52.

12. Hao C, Zhang G, Zhang L. Serum CEA levels in 49 different types of cancer and noncancer diseases. Prog Mol Biol Transl Sci. 2019;162:213-27.

13. Duffy MJ, Evoy D, McDermott EW. CA 15-3: uses and limitation as a biomarker for breast cancer. Clin Chim Acta. 2010;411:1869-74.

14. Ricci A, Mariotta S, Bronzetti E et al. Serum CA 15-3 is increased in pulmonary fibrosis. Sarcoidosis Vasc Diffuse Lung Dis Off J WASOG. 2009;26:54-63.

15. Moll SA, Wiertz IA, Vorselaars AD et al. Serum biomarker CA 15-3 as predictor of response to antifibrotic treatment and survival in idiopathic pulmonary fibrosis. Biomark Med. 2020;14:997-1007.

16. Kanazawa H, Yoshikawa T, Yamada M et al. CYFRA 21-1, a cytokeratin subunit 19 fragment, in bronchoalveolar lavage fluid from patients with interstitial lung disease. Clin Sci Lond Engl 1979. 1998;94:531-5.

17. Vercauteren IM, Verleden SE, McDonough JE et al. CYFRA 21.1 in bronchoalveolar lavage of idiopathic pulmonary fibrosis patients. Exp Lung Res. 2015;41:459-65.
18. Miyazaki K, Kurishima K, Kagohashi K et al. Serum KL-6 levels in lung cancer patients with or without interstitial lung disease. J Clin Lab Anal. 2010;24:295-9.

19. Kohno N, Akiyama M, Kyoizumi S, Hakoda M, Kobuke K, Yamakido M. Detection of soluble tumor-associated antigens in sera and effusions using novel monoclonal antibodies, KL-3 and KL-6, against lung adenocarcinoma. Jpn J Clin Oncol. 1988;18:203-16.

20. Yoshimasu T, Oura S, Ota F et al. Serum KL-6 Levels in Patients with Lung Cancer. J Pulm Respir Med. 2012;2:118.

21. Kamiya K, Watanabe M, Kohno M et al. KL-6 and CEA levels in epithelial lining fluid microsamples predict response to gefitinib in patients with advanced non-small cell lung cancer. Respirol Carlton Vic. 2011;16:976-82.

22. Yokoyama A, Kondo K, Nakajima M et al. Prognostic value of circulating KL-6 in idiopathic pulmonary fibrosis. Respirol Carlton Vic. 2006;11: $164-8$.

23. Kohno N, Hornada H, Fujioka S, Hiwada K, Yamakido M, Akiyama M. Circulating Antigen KL-6 and Lactate Dehydrogenase for Monitoring Irradiated Patients with Lung Cancer. Chest. 1992;102:117-22.

24. Briassoulis G, Stefanaki K, Peristeri I, Endoh Y, Hatzis T. Is KL-6 a serum indicator of early pulmonary fibrosis in childhood cancer patients? Med Pediatr Oncol. 2003;40:44-7.

25. Kodama T, Satoh H, Ishikawa H, Ohtsuka M. Serum levels of CA19-9 in patients with nonmalignant respiratory diseases. J Clin Lab Anal. 2007;21 103-6.

26. Balestro E, Castelli G, Bernardinello N et al. CA 19-9 serum levels in patients with end-stage idiopathic pulmonary fibrosis (IPF) and other interstitial lung diseases (ILDs): Correlation with functional decline. Chron Respir Dis. 2020;17:1479973120958428.

27. Prieto De Paula JM, Mayor Toranzo E, Gallardo Borge L, Franco Hidalgo S. Small-cell lung cancer and elevated CA 19.9 tumor marker levels. Arch Bronconeumol. 2012;48:385-6.

28. Dal Bello MG, Filiberti RA, Alama A et al. The role of CEA, CYFRA21-1 and NSE in monitoring tumor response to Nivolumab in advanced nonsmall cell lung cancer (NSCLC) patients. J Transl Med. 2019;17:74.

29. Mauro C, Passerini R, Spaggiari L et al. New and old biomarkers in the differential diagnosis of lung cancer: Pro-gastrin-releasing peptide in comparison with neuron-specific enolase, carcinoembryonic antigen, and CYFRA 21-1. In J Biol Markers. 2019;34:163-7.

30. d'Alessandro M, Bergantini L, Torricelli E et al. Systematic Review and Metanalysis of Oncomarkers in IPF Patients and Serial Changes of Oncomarkers in a Prospective Italian Real-Life Case Series. Cancers. 2021;13:539.

31. Goyette J, Geczy CL. Inflammation-associated S100 proteins: new mechanisms that regulate function. Amino Acids. 2011;41:821-42.

32. Hara A, Sakamoto N, Ishimatsu $\mathrm{Y}$ et al. S100A9 in BALF is a candidate biomarker of idiopathic pulmonary fibrosis. Respir Med. 2012;106:571-80.

33. Koh HM, An HJ, Ko GH et al. Prognostic Role of S100A8 and S100A9 Protein Expressions in Non-small Cell Carcinoma of the Lung. J Pathol Transl Med. 2019;53:13-22.

34. Oremek GM, Sauer-Eppel H, Bruzdziak TH. Value of tumour and inflammatory markers in lung cancer. Anticancer Res. 2007;27:1911-5.

35. Dai $\mathrm{H}$, Liu J, Liang $\mathrm{L}$ et al. Increased lung cancer risk in patients with interstitial lung disease and elevated CEA and CA125 serum tumour markers. Respirol Carlton Vic. 2014;19:707-13.

36. Liu Y, Zhu M, Geng J et al. Incidence and radiologic-pathological features of lung cancer in idiopathic pulmonary fibrosis. Clin Respir J. 2018;12: $1700-5$.

37. Bennett D, Salvini M, Fui A et al. Calgranulin B and KL-6 in Bronchoalveolar Lavage of Patients with IPF and NSIP. Inflammation. 2019;42: $463-70$. 